\title{
Vertigo in childhood: a methodological approach
}

\author{
Dispenza $\mathrm{F}^{1}$, De Stefano $\mathrm{A}^{2}$ \\ U.O.C. Otorinolaringoiatria, Sec. Otologia e Otoneurologia-Ospedale S. Giovanni di Dio, ASP1 Agrigento, Italy. \\ francesco.dispenza@gmail.com
}

\begin{abstract}
Children complaints dizziness merit meticulous evaluation to differentially diagnose a vestibular disease. A syndrome mimicking certain classic signs and symptoms of adult vestibular disorders may be presents in children, such as benign paroxysmal positional vertigo, usually associated with aging. Benign paroxysmal vertigo, in which migraine is a manifestation, with sudden onset of dizziness is a rare peripheral vestibular disorder that is commonly ignored or misdiagnosed. This review covers the development of the diagnosis, evaluation and treatment approaches of vertigo of childhood, a valid support for physician that approach dizzy children (Ref. 25). Full Text in PDF www.elis.sk.

Key words: vertigo, childhood, pediatric, dizziness, benign paroxysmal vertigo.
\end{abstract}

Although the vestibular symptoms in childhood are very rare, the occurrence of equilibrium disturbance could represent a challenge and an alarming condition. Fried in 1980 reported that only 14 pediatric patients among 2088 complained disequilibrium as main symptom (1). Also Eviatar in 1977 described 24 patients with vertigo among its personal archive of 5000 pediatric patients after neurological examination (2). An epidemiologic study of school population revealed that in $15 \%$ a symptom like disequilibrium was present once at least (3).

As in adults, several diseases could cause vertigo or, more properly, the equilibrium disturbances. Alteration of eyesight, inner ear dysfunction, nervous system (central and/or peripheral) disease, and disturbance of locomotive apparatus could be the cause of the disequilibrium.

As true vertigo it should be considered only the sensation of rotation of the surrounding environments, with or without nausea and vomiting. These symptoms are generally secondary to a disorder of the vestibular system at variable location: labyrinth or its network of connection with proprioceptive system, eyesight and central vestibular nuclei. Most of diseases that in adults usually cause equilibrium disturbance (i.e. cerebrovascular disease, cervical osteoarthrosis) are rare or totally absent in childhood, when instead the vestibular system is developing rapidly. The vestibular system is one of the first projections of the central nervous system (CNS) to become functionally active in children. As in adults the history recording and the clinical examination are the basis to reach a proper diagnosis. The pediatric patients are more inclined to adapt itself to disequilibrium and to compensate quickly a unilateral deficit rather than adults (4).

${ }^{1}$ U.O.C. Otorinolaringoiatria, Sec. Otologia e Otoneurologia - Ospedale S. Giovanni di Dio, ASP1 Agrigento, Italy, and ${ }^{2}$ Dipartimento di Scienze Chirurgiche, Sperimentali e Cliniche - U.O. Otorinolaringoiatria, Università degli Studi "G. d'Annunzio" Chieti - Pescara, Italia

Address for correspondence: F. Dispenza, MD, Via Oreto 339, 90124 Palermo, Italia.
Although the connection between labyrinth, proprioceptive system, cerebellum, and eyesight apparatus are incomplete at the time of the birth, the postural adaptations start in the prenatal time. The inner ear reaches the definitive shape after 8 weeks of intrauterine life, and the mielinization of the vestibular pathways, which occurs after birth, starts about 10 weeks before the pyramidal tract. The children aged between 18 months and 3 years old assign the acquisition of locomotors skills to the eyesight system rather than to the labyrinth or to proprioceptive system. At scholar age (4-6 years old) the integration between labyrinth, locomotor apparatus and eyesight is in quick developing and expansion, giving an image of functional adaptation of CNS pathways. The differential diagnosis of the diseases that generate disequilibrium in the pediatric patients is very challenging. A proper sequence of clinical evaluation includes: otorhinolaryngological consultation, neurological examination, and ophthalmologic visit. The disorders of ocular vergency could determine disequilibrium in children with normal vestibular function.

The most frequent cause of pediatric vertigo is the benign paroxysmal vertigo followed by migraine associated vertigo (5). Also the otitis media is considered one of the most common causes of pediatric vertigo.

\section{Clinical Management}

The clinical history gives a lot of useful information to achieve a diagnosis and a proper treatment of dizzy patients. Unfortunately in the pediatric age is very difficult collect anamnestic data directly from the little patients, as consequence the clinical history will be defective. The parents could furnish some important detail about onset modality of vertigo and about behaviors of the child. However, the subjective data felt by the patients, often the basis to orientate diagnosis in adults, will be missed. To persevere with questions to the little patient is often more helpful than collect the sensations of an alarmed parent. 
Obviously the age of the patients is crucial in the achievement of a good anamnesis. In newborn and in very young child the description give by parents is often the only one resource.

A retard in locomotors skills development could be due to a bilateral vestibular dysfunction; subsequently the labyrinthine function should be investigated in case of abnormal motor ability. The nystagmus (Ny) often is noted by the parents and reported it in the history, as well as the temporary lose of consciousness or the alteration of behavior (absence) could orientate to epilepsy. Like in adults, the rotatory vertigo, associated with nausea and vomiting, indicate a peripheral dysfunction of vestibular system, on the other hand the more generic disequilibrium is a symptom of a systemic disease (i.e. cardiovascular, metabolic, respiratory, and neurological). Precedent head trauma should be ruled out in the history collection, as well as should be noted headache episodes. The presence of migraine in parents should be investigated to rule out a familiar migraine syndrome. Otological symptoms and signs as: hearing loss, hear fullness, tinnitus, otorrhea, have to be recorded and history of ear disease as well. The equilibrium disturbances persisting more than 12 hours are more likely due to a CNS disease. Some alteration of the gait with closed eyes is suggestive for a bilateral labyrinthine dysfunction; on the other hand a difficult in gait with normal light condition and with opened eyes is a common symptom of a cerebellar disease. The anamnesis has to be completed with the recording of drugs in use, often a missed part in pediatric dizziness evaluation.

The otoneurological examination of a pediatric patient complaining vertigo includes three steps: head and neck examination, vestibular clinical evaluation and instrumental tests.

The head and neck examination should focus the attention to otoscopy, pneumatic otoscopy and cranial nerves function evaluation. The Ny investigation is very important in otoneurological practice, in pediatric age particularly, although it could be physiologic and transitory in the first years after birth. Few Ny jerks can be elicited physiologically in extremely lateral gaze. To avoid the Ny inhibition by gaze fixation it is useful to use the Frenzel's lens. The execution of Dix-Hallpike maneuver is mandatory to reveal a positional Ny also in children; few second of latency, fatigability, and spontaneous interruption are signs of peripheral vestibular lesion. A proper muscular tone and right movements of cervical tract are index of normal functioning of vestibular and proprioceptive inputs; often a wrong position of the neck could be a sign of vestibular disease in the little patients. In the newborn may be elicited the asymmetric tonic reflex of the neck: it consists in taking a characteristic postural scheme after head rotation, in particular the arm on the side of the face is extended, and the other one is flexed, determining the typical posture of a fencer. To evaluate the answer to the gravitational acceleration of the otoliths it is useful to do the vertical acceleration test (Moro's reflex); the normal response will be a superior limbs extension and abduction after a gravitational acceleration. The semicircular canal stimulation may be obtained also by rotation of the baby being in vertical position with the head $30^{\circ}$ flexed toward the side to investigate; in newborn could be noted the "eye-doll" movement. A Ny with rapid phase toward the rotation direction could be normally seen in the first 3 weeks of life. A vestibular dysfunction could be revealed with the postural adjustment test that consists in the superior limbs extension to prevent the fall on the same direction of a thrust. This maneuver should be executed with closed eyes to isolate the vestibular system and the response is present after 6 months of life and could be inhibited voluntarily after $2-4$ years old.

\section{Instrumental Examination}

The hearing threshold examination is mandatory because of $50-95 \%$ of pediatric patients with either acquired or congenital hearing loss have an associated vestibular dysfunction. The instrumental audiological tests are: behavioral audiometry, auditory brainstem response, acoustic otoemissions, pure tone audiometry, impedenzometry. The vestibular caloric tests, besides of difficult execution, are less sensitive than adults because of the myelinization of vestibular pathways are not yet completed. The rotatory test with Ny registration may be a good alternative to the caloric tests, although both labyrinths will be stimulated by a rotation. Since the sixth week of life most of normal children show a Ny after caloric or rotatory stimulation; it is remarkable that the tests stimulate only the horizontal semicircular canal. The ABR could be used as screening examination for the retrocochlear diseases and in children in which behavioral audiometry is not feasible. The correlation between ABR and vestibular diseases is not yet demonstrated (6). A prolonged dizziness with not well-defined characteristics should be investigated with MRI or CT scan. The electroencephalogram is required in case of suspected epilepsy.

\section{Common Causes of Pediatric Vertigo}

\section{Benign Paroxysmal Vertigo}

The Benign Paroxysmal Vertigo (BPV) of childhood is very common, but rarely is diagnosed by general practitioner or pediatric practitioner, this because of they don't get used to vertigo. Firstly described by Basser in 1964 (7), was codified in 1987 when 113 cases were reported in literature (8). Even if initially was interpreted as atypical vestibular neuritis, the BPV seems to be a variant of most common pediatric migraine as suggested by Fenichel (9), but the clinical presentation is without headache, differentiating also from migraine associated vertigo. The more common age of presentation is between 2 and 12 years old. The head position is not influent to start the vertigo spells in case of BPV, differentiating from benign paroxysmal positional vertigo, typical in adults, in which it is possible to elicit vertigo with modification of head position. The benign paroxysmal positional vertigo is very rare in children.

The BPV patients have a familiarity for migraine, and they can develop migraine after some year from the start of vertigo spell, even in its absence.

The typical clinical presentation is predominated by paroxysmal vertigo lasting second to minutes that may be associated with nausea and vomiting. The little patients remain aware and there is not blackout, although the child may be disorientated and could be search a support to prevent a fall. The parents can note often a Ny during the vertigo spell. Pure tone audiometry and clinical vestibular examination are normal, because of the little patients is visited far from the vertigo episodes. Some transient alteration could be noted 
on electronystagmography. The $43 \%$ of patients affected by BPV have a familiar history of migraine (mother) and the $13 \%$ of children will develop migraine during the life(10). The natural history of BPV is the spontaneous regression of vertigo spells after the 12th year, any medical treatment showed benefits in prevention of vertigo crisis. The actual management suggested in BPV is observation and treatment of accompanying symptoms (nausea and vomiting).

\section{Complicated Otitis Media}

The middle ear pathology is very common in childhood. Between the symptoms of the middle ear diseases the vertigo should be investigated. The middle ear effusion could be the reason of vertigo produced by a mechanical action on the medial wall of the middle ear cleft, where the middle ear is in communication with inner ear throughout the round and oval windows. The resolution of vertigo is immediate after drainage of the tympanum. A labyrinthitis could complicate an infective process of the middle ear. The infection or the bacterial toxins may pass the round window membrane and diffuse into inner ear. The symptoms in such cases are vertigo and hearing loss. The infection may reach the inner ear also throughout the cochlear aqueduct in case of meningitis or encephalitis. The treatment is the eradication of the infection and a therapy to reduce the inflammation with antibiotics and steroids. The cholesteatoma (congenital or acquired) could be cause of vertigo because of erosion of the otic capsule; more often the lateral semicircular canal is involved first. In these cases the treatment is surgical.

\section{Meniere's Disease}

Episodic vertigo, fluctuating hearing loss and tinnitus could orientate for a Meniere's disease, firstly described in pediatric patients by Crowe (11). In the children, before 10 years old, is difficult to obtain a clinical history that may orientate for Meniere's disease. The instrumental examination is helpful to reveal the hearing loss, which is generally fluctuating.

\section{Trauma}

The vestibular symptoms may be present after head trauma and whiplash injury. If the trauma causes a temporal bone fracture the vertigo will be associated with sensorineural hearing loss. A simple labyrinthine concussion is characterized by vertigo, similar to those present in BPV, but without radiological signs of fracture. The patients could develop vertigo and disequilibrium that may continue for several days after trauma. In some patients, after the resolution of the acute phase, may persist a benign paroxysmal positional vertigo. The instrumental investigations could show a variable hearing loss, but more frequently it is present a normal hearing function. The caloric labyrinthine stimulations reveal often some reflex alteration of the involved side.

A whiplash injury generally may generate a vestibular syndrome with positional vertigo persisting for several months. In these patients the hearing is normal, as well as the labyrinthine function, however may be present a transitory tinnitus. The orthopedic collar reduce the discomfort induced by neck rotation and could reduce the dizziness caused by such movements.

\section{Discussion}

Vertigo in the pediatric age is a challenge due to different causes and studies supporting the value of thorough history in evaluation of vertiginous children are present in English literature. It is extremely troublesome for parents, so prompt differential diagnosis is important, also because some conditions are treatable and benign. There are a great many diseases and, in general, vertigo can be due to either peripheral or central pathologies or to some benign conditions. For example, in BPV the history is the fundament of diagnosis because all radiological and otoneurological examinations, including audiometry, ENG, and electroencephalography, are normal(12-14).

The peripheral pathologies, often accompanied by auditory symptoms, also include congenital anomalies (genetic syndromes, embryopathies), familial causes (i.e. familial vestibular areflexia) and acquired causes (viral or bacterial neuro-labyrinthitis, trauma, Meniere's disease, delayed endolymphatic hydrops, chronic otomastoiditis, cholesteatoma, ototoxic drugs, autoimmune diseases) (13). The presence/absence of auditory symptoms associated with vertigo due to peripheral lesions is of the utmost importance for differential diagnosis. The central pathologies also include congenital anomalies (Arnold-Chiari malformation, vestibular epilepsy), familial causes (benign paroxysmal torticollis of infancy, familial periodic ataxia-vertigo) and acquired causes (infratentorial tumours, trauma, encephalitis, toxins). Dizziness associated with migraine may start in childhood or later in adulthood. The manifestations vary a lot ranging from episodic vertigo, to persisting disequilibrium, or motion discomfort. The vestibular symptoms can occur with or without headache, or before the onset of headache. Vertigo is sometimes the only symptom associated with the headache. Correct diagnosis is important to avoid inappropriate medical and surgical treatment of peripheral labyrinthine disorders (Meniere's disease for example) when they are not present(15-17). Previous studies have concentrated either on school aged children only or on children who were patients in ENT or neurological clinics, thus not revealing the true prevalence of vertigo in children (17). Children with peripheral vertigo are usually referred to an ENT clinic, while children with suspected central disorders are referred to a neurological clinic; the perspective of both neurologist and otolaryngologist may affect the selection in studies.

As stated before, in pediatric age, the most common cause of episodic vertigo without ear disease or hearing loss is BPV, whereas the BPPV, other benign condition, is rarely reported in children. BPV is characterized by brief attacks of incapacitating vertigo and postural imbalance: in absence of provoking factors, an otherwise normal child suddenly looks frightened, pale, sweaty; after 1-2 min, the child can resume all his/her activities. Auditory symptoms are typically absent and the attacks never induce loss of consciousness. Follow-up studies of patients affected with BPV confirm the hypothesis of a 'migraine equivalent and/ or precursor'. Brain stem involvement seems to be very frequent in paediatric migraine where initial attacks are often preceded by a history of hyperactivity in the first six months of the life, cyclic vomiting, recurrent abdominal pain, motion sickness and benign paroxysmal torticollis in infancy. 
Differential diagnosis should also include BPPV, the most frequent labyrinthopathy in humans, although it is rare in children. BPPV has a variable etiology (18) and is caused by a massive detachment of otoliths from the utricular macula.

The pathogenesis can be traced either to a direct ('cupulolithiasis') (19) or to an indirect action on the cupula ('canalithiasis') (20) generated by the gravitational shift of the otolith mass that occurs with a movement on the vertical plane. The clinical features are dominated by brief and intense vertigo of sudden onset, that is provoked by changes in head position. The most common provocative movements are lying down or getting out of bed, head hyperflexion and hyperextension. The other important symptom is the prolonged instability that can persist even after the disappearance of vertigo. As already reported, the Dix-Hallpike test is the classic diagnostic maneuver (21). The principal therapy is the removal of the mass through the non-ampullar hemicanal with the Semont maneuver or with one among the so-called repositioning maneuvers (22). BPPV must be correctly recognized because it can be efficaciously and rapidly treated. The overall success rate at the first maneuver is reported to be $80 \%$ (23). In our sample, vertigo disappeared at the first maneuver in all cases. Unsteadiness persisted for six days just in one case probably due to persisting macular symptoms.

Balance disturbances may occur also in children with otitis media. Serous labyrinthitis is responsible for vestibular disturbance (24). According to some authors, postural instability during otitis media with effusion is due to pressure changes in the middle ear cleft that results in labyrinthine symptoms through the action on round and oval windows (25). The insertion of ventilation tubes resolves most of the balance problems in both cases and early operative intervention is essential (25). Vertigo symptoms can be resolved by treating otitis media with antibiotics and in some cases also with a single paracenthesis. However, the main challenge is to identify the children with true vertigo from the child population with dizziness. Children reporting, for example, orthostatic hypotension or dizziness after alcohol drinking are quite easy identifiable and furthermore excluded from some study started.

\section{Conclusions}

Vertigo in childhood may be a challenge for all clinicians. The first address of the disease have to be made after a clinical examination, feasible, without instruments, by every physician. A multidisciplinary approach is, furthermore, mandatory to reach a right diagnosis and to start an appropriate treatment. The common causes of vertigo in children should be known to make differential diagnosis, and to reduce inappropriate instrumental examination. Most of the treatments are office-treatment or simple observation, managing only the acute symptoms.

\section{References}

1. Fried MP. The evaluation of dizziness in children. Laryngoscope 1980; 90: $1548-1560$.

2. Eviatar L, Eviatar A. Vertigo in children: differential diagnosis and treatment. Pediatrics 1977; 59: 833-838.
3. Russell G, Abu-Arafeh I. Paroxysmal vertigo in children: an epidemiological study. Int J Pediatr Otorhinolaringol 1999; 49: S105-S107.

4. Shirabe S. Vestibular neuronitis in childhood. Acta Otolaryngol 1988; 458: S120-S122.

5. Niemensivu R, Pyykko I, Kentala E. Vertigo and imbalance in children: a retrospective study in a Helsinki University otorhinolaryngology clinic. Arch Otolaryngol Head Neck Surg 2005; 131: 996-1000.

6. Brookhouser PE, Cyr DG, Peters JE, Schulte LE. Correlates of vestibular evaluation results during the first year of life. Laryngoscope 1991; 101: 687-694.

7. Basser LS. Benign paroxysmal vertigo of childhood (a variety of vestibular neuronitis). Brain 1964; 87: 141-152.

8. Finkelhor BK, Harker LA. Benign paroxysmal vertigo of childhood. Laryngoscope 1987; 97: 1161-1167.

9. Fenichel GM. Migraine as a cause of benign paroxysmal vertigo of childhood. J Pediatr 1967; 71: 114-115.

10. Parker W. Migraine and the vestibular system in childhood and adolescence. Am J Otol 1989; 10: 364-371.

11. Crowe SJ. Meniere's disease: study based on examination made before and after intracranial division of the vestibular nerve. Medicine 1938; 17: 1-36.

12. Al-Twaijri W, Shevell M. Pediatric migraine equivalents: occurence and clinical features in practice. Pediatr Neurol 2002; 26: 365-368.

13. D`Agostino R, Tarantino V, Melagrana A, Taborelli G. Otoneurologic evaluation of child vertigo. Int J Pediatr Otorhinolaryngol 1997; 40: 133-139.

14. Lanzi G, Balottin U, Fazzi E, Tagliasacchi M, Manfrin M, Mira E. Benign paroxysmal vertigo of childhood: a long-term follow-up. Cephalalgia 1994; 14: 458-460.

15. Harker LA, Rassekh CH. Episodic vertigo in basilar artery migraine. Otolaryngol Head Neck Surg 1987; 96: 239-250.

16. Weisleder P, Fife TD. Dizziness and headache: a common association in children and adolescents. J Child Neurol 2001; 16: 727-730.

17. Bower CM, Cotton RT. The spectrum of vertigo in children. Arch Otolaryngol Head Neck Surg 1995; 121: 911-915.

18. Parnes LS, Agrawal SK, Atlas JT. Diagnosis and management of benign paroxysmal positional vertigo (BPPV). CMAJ 2003; 169: 681-693.

19. Schuknecht HF. Cupulolithiasis. Arch Otolaryngol 1969; 90: 765-778.

20. Parnes LS, Mc Clure JA. Free-floating endolymph particles: a new operative finding during posterior semicircular canal occlusion. Laryngoscope 1992; 102: 988-992.

21. Dix MR, Hallpike CS. Pathology, symptomatology and diagnosis of certain common disorders of the vestibular system. Ann Otol Rhinol Laryngol 1952; 61: 987-1016.

22. Riggio F, Dispenza F, Gallina S, Kulamarva G, Gargano R, Speciale R. Management of Benign Paroxysmal Positional Vertigo of Lateral Semicircular Canal by Gufoni's Manoeuvre. Am J Otolaryngol 2009; 30: 106-111.

23. Dispenza F, De Stefano A, Mathur N, Croce A, Gallina S. Benign paroxysmal positional vertigo following whiplash injury: a myth or a reality? Am J Otolaryngol 2011; 32 (5): 376-380.

24. Golz A, Netzer A, Angel-Yeger B, Westerman T, Gilbert LM, Joachims HZ. Effects of middle ear effusion on the vestibular system in children. Otolaryngol Head Neck Surg 1998; 119: 695-699.

25. Grace ARH, Pfleiderer AG. Dysequilibrium and otitis media with effusion: what is the association? J Laryngol Otol 1990; 104: 682-684.

Received November 13, 2010. Accepted December 13, 2011. 